This item was submitted to Loughborough's Research Repository by the author.

Items in Figshare are protected by copyright, with all rights reserved, unless otherwise indicated.

\title{
How effective is the integration of sport and exercise medicine in the English national health service for sport related injury treatment and health management?
}

\section{PLEASE CITE THE PUBLISHED VERSION}

https://doi.org/10.23736/S0022-4707.18.08389-5

\section{PUBLISHER}

Edizioni Minerva Medica

\section{VERSION}

AM (Accepted Manuscript)

\section{PUBLISHER STATEMENT}

This work is made available according to the conditions of the Creative Commons Attribution-NonCommercialNoDerivatives 4.0 International (CC BY-NC-ND 4.0) licence. Full details of this licence are available at: https://creativecommons.org/licenses/by-nc-nd/4.0/

\section{LICENCE}

CC BY-NC-ND 4.0

\section{REPOSITORY RECORD}

Pullen, Emma, Dominic Malcolm, and Patrick Wheeler. 2019. "How Effective Is the Integration of Sport and Exercise Medicine in the English National Health Service for Sport Related Injury Treatment and Health Management?". figshare. https://hdl.handle.net/2134/33828. 
How effective is the integration of Sport and Exercise Medicine in the English National Health Service for sport related injury treatment and health management?

Emma Pullen, ${ }^{1 *}$ Dominic Malcolm, ${ }^{2}$ Patrick Wheeler ${ }^{3}$

${ }^{1}$ Faculty of Management, Bournemouth University, Bournemouth, UK; ${ }^{2}$ School of Sport, Exercise and Health Sciences, Loughborough University, Loughborough, UK; ${ }^{3}$ School of Sport, Exercise and Health Sciences, Loughborough University, Loughborough, UK.

*Corresponding author: Emma Pullen, Faculty of Management, Bournemouth University, Bournemouth, UK 


\begin{abstract}
Background: Regular participation in sport, exercise and physical activity is associated with positive health outcomes and form a mainstay of British public health policies. However, regular participation in sport and exercise can result in sport related injury (SRI) which, in turn, is a key cause of exercise cessation. The integration of Sport and Exercise Medicine (SEM) in the English National Health Service (NHS) aims to provide a specialist service for public populations and thus reduce the impact of SRI on exercise cessation and associated negative health outcomes. More broadly it aims to both support physical activity health promotion policies and improve healthcare organisations efficiencies through providing the most condition-appropriate treatment.
\end{abstract}

Methods: This qualitative interview study examines patients' $(n=19)$ experiences of accessing and receiving SEM treatment within the English NHS.

Results: The research demonstrates that referral pathways into SEM were often prolonged, characterised by multiple General Practitioner (GP) visits and referrals into other musculoskeletal services, demonstrating an inefficient use of healthcare resources. Prolonged pathways fostered only limited recovery back to previous physical activity levels and other negative health behaviours, yet on accessing the SEM clinic, patients experienced progressive rehabilitation back into sport and exercise participation.

Conclusion: This study highlights the importance of more fully integrating SEM services into public healthcare as a way of improving the organisational capacity of healthcare in treating SRI and ensuring that citizens comply with state interventions which orchestrate health management through raising physical activity levels across the population.

Key Words: Sport Injury, Sport and Exercise Medicine, NHS, Health, Policy 


\section{Introduction}

Participation in sport, exercise and physical activity (PA) is associated with a significant reduction of cardiometabolic risk, ${ }_{1-2}$ increased overall 'well-being' ${ }_{3}$ and is seen to contribute to positive health outcomes for the whole of society.4 The importance of activity for health management is indicated in the promotion of exercise as both preventative and restorative 'medicine'.5 Consequently, physical activity health promotion (PAHP) policy has grown in prominence and become increasingly 'politicised'. For example, a review of national documents published in the 28 EU member states between 2000 and 2009 identified 112 which mentioned 'health-enhancing physical activity and contained overall goals on participation in sport and physical activity and/or on health promotion'.6 Despite a desire to encourage even small increases in physical activity, the health benefits are believed to be greater in relation to the consumption of more vigorous and intense forms of activity.7 Consequently, PAHP policies deliver health messages that iterate the uptake of both moderate forms of physical activity (e.g. walking) and intense, vigorous exercise, such as running and football.4

Whilst sport, exercise and PA can provide health promoting benefits, critics suggest that the evidence base for the PAHP movement entails a conflation of science and politics.8 This is typified through the ontological equation of the health benefits of PA and sport with little, if any, recognition of the wider social, cultural and organisational contexts that impact the efficacy of sport and exercise in the promotion of population health outcomes. For instance, estimates of the proportion of national populations incurring sport related injury (SRI) each year range from 3.1\% in Germany,9 to 5.9\% in Australia,10 8.1\% in England and Wales 11 and 10.1\% in Canada.12 The most comprehensive British epidemiological study to date 11 estimated that each year there were 29.7 million SRIs, with the highest incidence rate occurring in 
'vigorous sports' that allow contact (such as football) and 'intrinsic' activities (i.e. entailing no outside object or person) such as running. Moreover, with any form of sport or exercise, the risk of sustaining a SRI is related to the increasing frequency, type of activity, nature, and intensity of activity.13 Not only does this detail conflict with the universally projected positive health outcomes of sport and exercise, but it remains largely absent from PAHP messages which are underscored by a 'more is better' portrayal of sport and exercise participation. SRI's are a major cause of morbidity 14 and this has been documented by epidemiological studies conducted in a variety of developed countries.15-17

A further impact of SRI on population health is through enforced exercise cessation 18 and a reduction in the return to both vigorous and more moderate forms of activity following SRI.19 Evidence further documents that those whose sport and exercise participation is restricted due to injury are unlikely to 'compensate' with more moderate forms of activity, and are therefore likely to withdraw from all forms of exercise post injury.19 Whilst there may be multiple contextual factors influencing this, the disruption of SRI to the structured, habitual (and thus long-term health promoting) participation in regular sport and exercise has been noted as one factor. 20

However, where SRI is sustained, medical treatment is often sought.17 In response to this, Sport and Exercise Medicine (SEM) services, designed to deliver specialist SRI treatment and rehabilitation for public populations, have been integrated into the National Health Service (NHS) in England. The establishment of SEM services is part of a broader health management strategy which aims to facilitate populations' continued uptake of sport, exercise and Physical Activity (PA) and associated positive health outcomes, while also contributing to the organisational capacity of the health service in treating SRI. Therefore, the purpose of this study was to explore patients' experiences of utilising SEM for SRI treatment, their pathways through NHS services to these clinics, and the efficacy of this service, particularly in the 
treatment and management of SRI for continued participation in sport and exercise. A description of the role and initiatives of SEM in the NHS are detailed below to provide a contextual backdrop to the study.

\section{Sport and Exercise Medicine in NHS England}

The scale of SRI noted above places a significant burden on public health services. For instance, SRIs are commonly presented to, and account for, a substantial proportion of primary care consultations, creating a workload for both family/general practitioners (GPs) and emergency care.21 The subsequent onward referral and transition of patients into orthopaedic secondary care for specialist treatment mean that these services receive more than two million referrals a year and frequently have particularly long waiting times.22 Organisational inefficiencies often lead to sub-optimal injury management, exacerbate exercise cessation, and subsequently contribute to poor health for these population groups.21

The introduction of SEM in NHS England was expected to improve the capacity and efficiency of musculoskeletal services across primary and secondary care and thus reduce the subsequent exercise cessation and associated poor health outcomes linked to SRI.23 For instance, in relation to secondary care, SEM clinics were established to reduce the cumulative pressures on existing orthopaedic services (e.g. non-SEM outpatient physiotherapy) by providing 'a single point of referral for patients' where the integration of combined services (e.g. radiology, podiatry and specialist SEM physiotherapy) provide diagnostics, treatment, and rehabilitation of SRI. This integrated approach is therefore focused on the non-surgical medical management of SRI,23 providing specialist SRI treatment that intends to rehabilitate patients back to previously seen physical activity levels. Whilst improving the medical management of SRI at the patient level is a key initiative of SEM's integration within the NHS, it is expected to contribute to the overall capacity to treat SRI and thus improve the efficiency of treatment at the organisational 
level. Key benefits include improving the transition of patients with a SRI through the NHS via initial and direct referrals into SEM. This is expected to subsequently reduce the burden placed on primary care, particularly in the utilisation of GPs and orthopaedic secondary care. Moreover, the integration of SEM aimed to increase efficiency through the integrated clinical management of SRI, reducing SRI patients' waiting times and transitions between services and, thus, improving the patient satisfaction with their healthcare experience. 23

Existing research on the integration of SEM in the NHS has documented a low awareness amongst healthcare practitioners (e.g. non-SEM outpatient physiotherapists and GPs) of the role of SEM within the NHS.24-25. A significant number of GPs (83\%) reported having never referred a patient to SEM services which, inevitably meant a lack of a standardised patient pathway.24 However, there remains no research that delineates this referral pathway in detail, or captures the perspective of the patients who receive treatment for SRIs both within and outside of SEM services. This study is therefore the first to explore the experiences of British NHS patients utilising SEM services for SRI treatment and rehabilitation. The extent to which SEM's integrated service approach is being experienced by patients and the effectiveness of SEM treatment and rehabilitation for patients with a SRI is critical to maximizing the potential benefit SEM provision has in facilitating continued exercise participation and wider health outcomes. Indeed, this is particularly important in a climate where increasing PAHP policy is focused on long term and structured participation in sport and exercise for all population groups, across the lifecourse.

Based on the existing research in this area we hypothesise that patient pathways into SEM services will be complex and unstandardized indicating the marginalisation and ineffective integration of SEM in NHS England. Therefore, the purpose of this study is to document patients' $(n=19)$ pathways to one SEM clinic in NHS England, the efficacy of the treatment 
received, and the impact of SEM treatment on their continued or resumed participation in sport and exercise.

\section{Materials and Methods}

The study adopted a qualitative research design that included a single semi-structured interview with participants receiving treatment at one SEM clinic in NHS England. Interviews took place over a period of 15 months. The study was granted NHS research ethical approval by NHS REC East Midlands-Nottingham 2 and NHS site specific approval at one hospital-based SEM clinic in NHS England. All NHS-based research was conducted in line with HRA guidance. REC reference: 14/EM/1191

Participants ( $\mathrm{n}=19)$ were recruited according to predefined inclusion and exclusion criteria. Inclusion criteria sought individuals who: (a) were being treated at the SEM clinic; (b) had sustained a physical injury as a result of participation in sport/exercise/PA practices; (c) had injuries categorised as chronic (defined as ongoing after 6 weeks of injury time); (d) were fluent English speakers; (e) were aged 18 or over. The exclusion criteria were based on: (a) patients unable to give informed consent; and (b) patients unable to attend initial discussion for a study, and/or subsequent face-to-face meetings. The inclusion and exclusion criteria allowed for patients to be relatively demographically diverse and provide as representative a sample of the general population as possible.

Patients who met the inclusion criteria were initially approached by the clinician treating their SRI at the SEM clinic and asked if they were interested in taking part in the study. Interested patients were then provided with a participant information sheet (PIS) outlining the study and the commitment required. Following this, the clinician recorded the contact details of all 
patients who had a PIS and passed this information to the principal investigator/first author. The researcher then contacted the patient after at least a minimum cool-off period of 24 hours to confirm continued willingness to participate and, if so, arrange a meeting at the SEM clinic. Within this meeting, informed consent was sought and an interview conducted. The details of 23 patients who met the inclusion criteria were recorded by clinicians and, of these, 19 were subsequently willing to be interviewed. Pseudonyms have been used to protect patient confidentiality.

A systematic literature review was conducted to inform the design of the qualitative interview schedule. Interviews lasted up to one hour and were conducted in a private room at the SEM clinic. All interviews were audio recorded and transcribed verbatim providing a written transcript for data analysis. Data was collected until the point of data saturation to enrich content validity.26

Data was analysed using thematic content analysis led by the principal investigator. This process included data familiarisation, extraction of common thematic units of text identified across patient transcripts through open coding, and organisation of codes into thematic clusters.27 Codes were cross-checked and 'critical friends' utilised to establish credibility of thematic codes. This included an open discussion of the thematic interpretations made from the data analysis with colleagues until an interpretive consensus was reached. Themes were labelled to represent the chronological pathways of patients. Below we discuss the four most pertinent. These are: Respective Roles of GPs and Secondary Care; Impact on Health; Transition to SEM; and Experience of SEM.

\section{Results}

\section{Respective Roles of GPs and Secondary Care}


Findings indicate that all patients had initially accessed the NHS through their General Practitioner (GP) or family doctor and were experiencing predominantly soft tissue injuries from overuse. However, of the 19 patients, only 2 had received an initial direct referral from their GP to the SEM clinic. The majority, therefore, were either prescribed pain medication and/or referred to orthopaedic secondary care services prior to being seen in the SEM clinic. Where patients utilised pain medication and/or orthopaedic care they experienced multiple GP consultations. This ranged from between two to five visits across the patient sample. For example, one patient, who sustained a hamstring injury (later diagnosed by the SEM consultant as Hamstring Tendinopathy) approximately a year before attending the SEM clinic, had visited his GP three times over approximately eight months before being referred to non-SEM outpatient physiotherapy.

I went back to the doctor and she gave me some codeine. I got used to the codeine and it killed my back pain [but] I went back to the GP for a third time before I finally got referred.

A further patient, who was experiencing pain in his Achilles tendon from running described multiple visits to his GP over a two-year period.

I might have seen four different GPs at that practice ... [they] didn't seem very interested in resolving the problem, just prescribed more painkillers ... I think each consultation was ten to fifteen minutes maybe with the GP which happened several times.

When GPs referred patients to secondary care, the most common initial referral pathway was into non-SEM outpatient physiotherapy services. For several patients this led to some improvement in the condition of their SRI. However, a large proportion of patients described outpatient physiotherapy as being ineffective, reporting that this treatment was not tailored to 
returning to their previously physically active state. This is demonstrated in the following extracts:

Nothing seemed to be working. I was up here [at the hospital] once every 2 or 3 weeks for a quick 5 minutes in with physiotherapy and [told] “just keep doing what you are doing” but there was no progression with fixing the injury.

He (GP) referred me for physiotherapy. I started there at the hospital, after 18 months I had plateaued.

It’s [physiotherapy] too generic. I told them my activity levels and how much I was working and they just said do the stretches 10 times a day and there was no progress.

Some patients described multiple GP referrals into the same secondary care service - typically non- SEM outpatient physiotherapy - despite expressing their dissatisfaction with the previous outcomes. This is illustrated in the extract below from a patient who had been experiencing back pain for up to 4 months.

I understand you've got to try with the physios first and try and get things under control a bit but it wasn’t going away so I went to the GP surgery again and they got me back with the same lady at physiotherapy... I thought if we know it won't solve the issue because it's not a physiotherapy issue, why am I going to physiotherapy?

\section{Impact on Health}

Patients who utilised outpatient physiotherapy and reported this service as ineffective in returning them to their previously physically active state, continued to experience limited exercise participation and, in some cases, total cessation. Despite the rehabilitation advice provided, many patients remained inactive for extended periods of time. For some patients, this led to claims to have 'given up on sport' as they remained inactive having previously engaged 
in regular and habitual exercise. They were also likely to be unaware of the alternative treatment options available to them. This is captured in the interview extracts below. For instance, one patient who had regularly participated in gym activities prior to sustaining a knee injury claimed:

I haven’t done any sport or exercise for over two years. Whenever I tried to it [SRI] was just coming back each time.

The patient who was experiencing pain in his Achilles tendon explained:

I think up until two years ago [prior to SRI] I was running regularly. The last time I ran in pain was probably about a year and a half ago and I have just sort of given up on that now.

One patient who had utilised outpatient physiotherapy for a second time was discharged but remained unable to participate in even moderate physical activity. He claimed

I walked into town the other day, right, its 2 miles and I got back hobbling. Couldn’t walk.

Many patients spoke of the direct impact of exercise cessation on their health, making claims to weight gain and general overall poor well-being. The extent of this impact on patients' perceived health varied depending on the length of time they remained in the NHS 'system' seeking effective treatment. For instance, one patient, who had been seeking treatment for approximately one year for an injury to her lower back claimed:

I’m noticing the knock-on from that [SRI] already. You know a potbelly. Fortunately, I'm very active in all other work that I do, but exercise-wise, I don't do any training for that now. 
Where patients have lived with SRI for a significant duration of time the impact of exercise cessation on wider health behaviours is potentially more serious. One patient who had been living with a knee injury for up to 2 years explained:

I used to be health conscious and active, now it's just fat and lazy. You end up binging on bad food. If you're not in that exercise regime then you get bad habits. The stress at work gets you into bad habits, eating late, eating rubbish... Not being able to do my hill walking has really hit me.

\section{Transition to SEM}

For the majority of interviewees, the transition from the GP or outpatient physiotherapy into the SEM clinic was indirect and did not appear to follow a standardised pathway. Consequently, patients' pathways were often needlessly lengthy. Throughout this time, patients remained largely inactive. Findings indicated that the majority of patients were unaware that specific SEM services were available, and that the majority of GPs did not directly and/or initially refer patients into the SEM clinic or discuss this treatment service. More frequently patients described learning about the service through their engagement with other secondary healthcare providers. For example, one patient reported being informed about the SEM clinic by a hospital receptionist before returning to her GP and requesting this service.

I was querying a mix up between my private healthcare, and the lady on the phone that

I was asking about the mix up said "oh have you heard of the sport medicine clinic?”

So I went back to my doctor and said, “do you know about the sport medicine clinic?” My doctor sent a letter off for and I finally got an appointment with a specialist.

This referral to the SEM clinic was therefore dependent on a serendipitous conversation and is indicative of a non-systematic service provision. There were further incidences of patients 
being referred largely by chance through interaction with locum doctors. For instance, the patient who was experiencing pain in his Achilles tendon (with a later diagnosis of Achilles Tendinopathy) and had visited his GP 4 times previously, described how the SEM provision was seemingly 'unheard of' before the meeting with the locum doctor, which suggests that access to SEM services in the NHS is as much the result of limited awareness as restricted supply.

I had no idea about SEM. When she [physiotherapist] discharged me from her care, I had no idea what options were left. So I didn't know whether the GP was just going to prescribe more painkillers or what. The saving grace is that I went back to the surgery and there was a locum there - so, a temporary [doctor] - and he felt my heel, said, "It's Achilles Tendinopathy," straight away, "We'll get it confirmed with an ultrasound”. Within four weeks I finally had a referral for the SEM clinic... and that's when this place came to light and everything sort of turned around.

Similarly, some patients reported learning of the SEM clinic whilst being treated in outpatient physiotherapy. When he raised this treatment option during a subsequent GP consultation, his GP appeared to lack knowledge on this service:

I had spoken to the physiotherapist who had told me about the sports clinic. I felt there is something more they can do, I didn't know what but the fact it had been mentioned I thought there is a next level and that [it] was available to me. I went back to the GP but I don’t think he knew it existed.

\section{Experience of SEM}

Once patients accessed the SEM clinic and were seen by a specialist they became highly satisfied with their treatment. One reason for this is patient awareness of an SRI management 
plan, achieved through diagnosis, treatment and specialist advice, which SEM's integrated service approach is designed to deliver.24 This significantly reduced the waiting times of patients between services and gave patients a greater sense of control and understanding over their treatment pathway contributing to both improved patient satisfaction and patient outcomes. For example, patients described the speed of their pathway compared to standard orthopaedic services:

They get you in quickly, I saw him [SEM consultant] only a couple of weeks ago and I am having my treatment Wednesday. I am moving in the right direction now. I was really disheartened before.

To be seen so quickly now and its moving along at a decent speed. He [SEM consultant] has gone through everything with me.

Patient satisfaction is further improved by the rehabilitation advice SEM consultants provided for their return to previous physical activity levels. This tailored advice contributes to preventing exercise cessation and provides patients with bespoke exercise and Physical Activity (PA) guidelines dependent on the nature of patients' conditions and aspirations. For patients, this led them to perceive a more meaningful progress towards recovery, plus a strategy for the longer-term continuation of exercise and wider health goals. For instance, this is described in the extract by a patient who had been experiencing a running injury to her lower back for up to 6 months of which was diagnosed once she was referred to the SEM clinic:

Once I got diagnosed at the SEM that was a big help. It put closure on it and I finally knew what I could and couldn't do... I could do a bit more walking but no running. But I had to gradually do it. So I had a gradual increase of what I was allowed to do. By the end of September after treatment I was allowed to start running. 
My aim is to do the Edinburgh Moonwalk. If things are going the way they are going I will be fit to get the training in and complete it.

Ultimately, patients view SEM as markedly more effective than the other healthcare services that they had encountered.

\section{Discussion}

The findings reported here highlight the role of SEM in managing SRI rehabilitation and reducing exercise cessation. It highlights the degree to which the effective management of SRI in public populations through specialist SEM services could potentially contribute to broader health management government agendas. These findings therefore begin to correct the existing knowledge deficit in relation to the integration of SEM and the experience of SEM treatment and rehabilitation for patients utilising this service.

Despite the positive patient experiences of SEM documented here, the findings demonstrate that there remain significant problems in the organisational delivery of SEM which restricts the degree to which such clinics contribute to government health goals through the management of SRI. Firstly, there remains a lack of awareness amongst both healthcare providers and patients of the provision and potential of SEM as a secondary care service in SRI treatment and rehabilitation. This substantiates previous research on SEM integration that demonstrates a low awareness of the role of SEM by GPs and multiple and non-standardised pathways.24 Secondly, however, these findings illustrate that longer established forms of treatment lead the majority of patients to experience relatively lengthy and therefore inefficient treatment pathways, with access into SEM often engendered by unintended consequences rather than more considered and purposeful action taken by healthcare practitioners. As a result of ad-hoc referral pathways, 
patients often visited their GP multiple times, mainly received pain relief, and were frequently referred by their GP into secondary care orthopaedic services which, they found, did not significantly relieve symptoms and provide a progressive recovery back into regular sport and exercise participation.

Following patients' access of SEM, satisfaction with treatment improved, facilitated by the single approach to clinical service integration and specialist exercise focused rehabilitation. This ultimately improved the speed of patient pathways and patient outcomes, with a significant number of patients returning to structured and regular physical activity and exercise. The specialist rehabilitative advice SEM clinicians were able to offer further improved patient satisfaction and, importantly, prevented exercise cessation which commonly results from suboptimal injury management of SRI and can lead to poor health behaviours.

Indeed, patients' experiences of exercise cessation illustrates how the impact of SRI on the continuing participation in sport and exercise could be ameliorated through more effective healthcare delivery. The findings presented here provide a qualitative, 'lived' insight into the experience of forced exercise cessation and how this in turn becomes entwined with other forms of 'unhealthy' living. It further highlights the importance of contextually relevant rehabilitative advice on exercise cessation/continuation for populations who participate in sport, exercise and PA and may have specific SRIs 'health' needs. SEM services appear to be highly effective in enabling the return to regular and structured activity levels among this population and the value of this kind of tailored advice is made all the clearer by patients' perceptions of the organisational efficiencies of other healthcare services. Whilst the inconsistent, incoherent and inefficient pathways mean that invariably delays happen across a patient journey leading to unnecessary utilisation of healthcare resources, specifically in services that already see high demand, they further reinforce the compounded negative impact SRI symptoms have on individuals' levels of physical activity, sport and exercise participation. 
It is therefore evident that the underlying goal for integrating SEM services in the English NHS has yet to be fully achieved and the full utility of SEM in the NHS remains unfulfilled. This raises a number of important implications for the sustainability of SEM services, PAHP policy, and the successful facilitation of improved population health.

\section{Implications}

If the full utility of SEM in the NHS is to be realised, improved knowledge amongst both GPs and patients on the role and function of this service is required. This would not only contribute to lessening exercise cessation among patients with SRIs by reducing some of the existing inefficiencies in patient pathways into SEM, but further improve the organisational capacity of the broader healthcare system by reducing the number of repeat GP consultations by SRI patients and the subsequent and often ineffective use of secondary care. This is a particularly important implication for GPs given their position in health management as 'gatekeepers' to secondary healthcare services. GPs are a critical group in implementing the strategic initiative of providing 'a single point of referral for patients requiring specialist help’.23

Moreover, these findings have important implications for the sustainability of government policies which iterate the importance of less sedentary lifestyles and more vigorous forms of sport, exercise and PA. Increased participation entails an increased potential injury burden, and injury is frequently cited as a primary reason for exercise cessation.19 The longer and more protracted patient pathways into SEM are, the more likely it is that those experiencing SRI will either reduce their physical activity levels and/or withdraw from exercise altogether. As these findings show, forced exercise cessation can have a negative impact on health behaviours and well-being as inactivity spirals into poor diet, and thus potentially become a greater burden on health services in the future. Thus, whilst exercise can be a form of "medicine" ${ }_{5}$ for population health it is increasingly important to recognise the capacity of sport and exercise to create 
population ill-health. The benefits of sport and exercise for population health can only be achieved if there is a wider acknowledgement of the multiple contextual factors that make the delivery of sport an effective tool for PAHP.

Governments must therefore explicitly recognise the inherent risk of sustaining SRI that more vigorous forms of activity entail and develop parallel strategies to deal with an injury burden that will inevitably evolve from successful exercise promotion. This paper has demonstrated the as yet unfulfilled role of SEM as one potential parallel strategy and the potential role it could have in promoting continued and regular participation in sport and exercise. However, if SEM remains a relatively marginalised aspect of health management then there will continue to be relatively significant inefficiencies in the management of SRI across the public and the goal of achieving a more active, healthier, population, will be thwarted.

\section{Reflections}

The patient pathways described here stem from one patient group utilising one SEM clinic in NHS England. Due to the recruitment bias inherent to the location of data collection, this study represents the experiences of patients whose SRIs were not effectively treated elsewhere, e.g. by GPs via non-SEM outpatient physiotherapy services. We recognise that it is likely that a large proportion of SRI patients can achieve a full recovery without SEM intervention and thus suggest that the findings are indicative rather than generalizable. Secondly, this study deals with a relatively small sample, although we note that its size is appropriate for the qualitative design of this study and that data saturation was achieved.26 Knowledge from this study can be used to improve the efficiency of pathways for SRI patients into SEM clinics and the wider integration and utility of this service as an effective parallel strategy of SRI prevention in public population groups.28 We recommend that further research be conducted in this area, including that which focuses on the complexities of direct and initial referral for patients with a SRI by 
GPs. This is required to gain a greater understanding of the organisational barriers to improving patient pathways into SEM services. This will be integral for the longer-term sustainability of government PAHP that aims to increase the numbers of public populations participating in sport for improved public health.

\section{References}

1. Kohl, HW III. Physical activity and cardiovascular disease: evidence for a dose response. Med Sci Sports Exerc 2001;33:472-83.

2. Tune I, Furberg AS. Physical activity and cancer risk: dose-response and cancer all sites and specific sites. Med Sci Sports Exerc 2001;33:530-50.

3. Hassmén P, Koivula N, Uutela A. Physical exercise and psychological well-being: a population study in Finland. Prev Med 2000;30:7-25. 
4. Public Health England. Health Matters: Getting Every Adult Active Every Day. https://www.gov.uk/government/publications/health-matters-getting-every-adult-activeevery-day/health-matters-getting-every-adult-active-every-day 2016. Accessed 31 August 2017

5. Sallis RE. Exercise is Medicine and physicians need to prescribe it! Brit J Sports Med 2009;43:3-4.

6. WHO. Promoting Sport and Enhancing Health in European Union Countries: a policy content analysis to support action. [Internet]World Health Organization. Europe: Copenhagen. 2011:42

7. Swain DP, Franklin BA. Comparison of Cardioprotective benefits of vigorous verses moderate intensity aerobic exercise. Am J Cardiol 2006;97:141-7.

8. Bercovitz K. ‘A critical analysis of Canada’s “Active Living”: science or politics?’. Crit Public Health 2000;10:19-39.

9. Schneider S, Seither B, Tonges S, Schmitt H. Sports injuries: population based representative data on incidence, diagnosis, sequelae, and high risk groups. Brit J Sports Med 2006;40:334-339.

10. Egger G. Sport injuries in Australia: causes, cost and prevention. Health Promot J Austr $1991 ; 1: 28-33$

11. Nicholl JP, Coleman P, Williams BT. The epidemiology of sports and exercise related injury in the United Kingdom. Brit J Sports Med 1995;29:232-238 
12. McCutcheon T, Curtis J, White P. The socio-economic distribution of sport injuries: multivariate analyses using Canadian national data. Sociol Sport J 1997;14:57-72 13. Verhagen EALM, van Mechelen W. Sport for all, injury prevention for all. Brit J Sports Med 2010;44:158-158.

14. Baker R. Randomised controlled trial to compare GP - run orthopaedic clinics based in hospital outpatient departments and general practices. Br J Gen Pract 2005; 55:908-10

15. Baarveld F, Visser, CA, Kollen BJ, Backx FJ. (2011). Sports-related injuries in primary health care. J Fam Pract 2011;28:29-33.

16. Dekker R, Van Der Sluis CK, Groothoff JW, Eisma WH, Ten Duis HJ. Long term outcome of sports injuries: Results after inpatient treatment. Clin Rehabil 2003;17:480-487.

17. Grice A, Kingsbury SR, Conaghan PG. Nonelite exercise-related injuries: Participant reported frequency, management and perceptions of their consequences. Scand J Med Sci Sports 2014;24:86-92.

18. Finch C, Owen N, Price R. Current injury or disability as barriers to being more physically active. Med Sci Sports Exerc 2001;33:778-782.

19. Andrew NE, Wolfe R, Cameron P, Richardson M, Page R, Bucknill A, Gabbe B. The impact of sport and active recreation injuries on physical activity levels at 12 months postinjury. Scand J Med Sci Sports 2014;24:377-385.

20. Rhodes RE, De Bruijn GJ. Automatic and motivational correlates of physical activity: does intensity moderate the relationship? Behav Med 2010;36:44-52.

21. Batt M, Cullen M. Sport and exercise medicine in the United Kingdom comes of age. BMJ 2005;39:250-251. 
22. Ferguson S, Cook F. Is a primary care orthopaedic interface service sustainable in a continually changing political and healthcare environment? Clin Gov 2011;16:137-147.

23. Jones NSC, Weiler R, Hutchings K, Stride M, Adejuwon A, Baker P, Larkin J, Chew S. National Health Service: Sport and Exercise Medicine: A Fresh Approach http:/ / www.fsem.co.uk/ DesktopModules/ Documents/ DocumentsView.aspx?tabID=0\&ItemID=1 15345\&MId=5288\&wversion=Staging] 2012;Accessed 7 July 2015.

24. Kassam H, Brown VT, O’Halloran O, Wheeler P, Fairclough J, Maffulli N, Morrissey D. General practitioners' attitude to sport and exercise medicine services: a questionnaire-based survey. Postgrad Med 2014;90:680-684.

25. O'Halloran P, Tzortzious Brown V, Morgan K, Maffulli N, Perry M, Morrissey D. The role of the sports and exercise medicine physician in the national health service: a questionnaire-based survey Brit J Sports Med 2009;43:1143-1148.

26. Bowen GA. Naturalistic inquiry and the saturation concept: A research note. Qual Res 2008;8:137-152.

27. Braun V, Clarke V. Using thematic analysis in psychology. Qual Res Psychol 2006;3:77101.

28. Finch CF. Getting sports injury prevention on to public health agendas - addressing the shortfalls in current information sources. Br J Sports Med 2012;46:70-74 
